\title{
EVOLUCIÓN, ESPACIO Y ADAPTACIÓN EN GRUPOS CAZADORES-RECOLECTORES
}

José Luis Lanata*

\begin{abstract}
LANATA, J.L. Evolución, espacio y adaptación en grupos cazadores-recolectores . Rev do Museu de Arqueologia e Etnologia, S. Paulo, 3: 3-15, 1993.
\end{abstract}

RESUMO: Neste artigo são discutidos o uso de espaço entre caçadorescoletores e a relação com a adaptação geral e evolução. Os arqueólogos frequentemente vêem a distribuição de artefatos similares no espaço como pedaços de territórios humanos, sem nenhum tipo de suporte metodológico que sustente isso. A visão etnográfica na explicação do registro arqueológico está escondida na variabilidade de respostas humanas aos diferentes meios (natural, social, etc.). Este artigo mostra que a idéia de território em arqueologia necessita ser posta de lado e ser usada numa abordagem mais consistente que atinge com maior precisão as propriedades do registro arqueológico.

UNITERMOS: Análise espacial - Caçadores-coletores - Adaptação.

\section{Introducción}

En las últimas dos décadas, los enfoques teóricos en la arqueología de los grupos cazadoresrecolectores han sido sustancialmente modificados con la incorporación de la etnoarqueología y los enfoques evolutivos y ecológicos, entre otros.

El impacto de estas nuevas perspectivas, conjuntamente con el reanálisis del status de la arqueologia como ciencia (i.e. Clarke, 1968; Flannery, 1977; Binford,1977) hace necesario poder conjugar la naturaleza del dato arqueológico con la posibilidad de contar con una teoría general como un medio para poder comprender la conducta humana. Este puente es conocido como "Middle Range Theory" - teoria del rango medio - por varios autores (ver Raab and Goodyear, 1984 para una compilación; Thomas, 1986; Grayson, 1986; confrontar con Schiffer, 1988). La necesidad

(*) Dep. de Antropologia Uni. de Buenos Aires y TARL Uni. of Texas at Austin. de emplear una teoria general - o de desarrollar una de rango medio - generó además, un sinnúmero de acercamientos distintos - sean estos metodológicos o técnicos. La variedad de aproximaciones a los sistemas de cazadoresrecolectores (Jochim, 1979; Bettinger, 1980 y 1991; Dunnell, 1980 y 1989; Smith y Winterhalder, 1981 y 1992; Thomas, 1983; Hodder, 1986; Schiffer, 1987; Smith, 1991, etc.) nos muestran lo difícil que puede, ser tratar unificar en una teoria general las diferentes perspectivas disponibles actualmente. Sin embargo, Dunnell (1992 a y b) es uno de los pocos que ha sugerido que la teoría de la evolución puede ser un marco teórico general aplicable a la arqueología (ver también Dunnell 1980, Rindos 1984, Leonard y Jones 1987).

\section{Espacio, territorio y territorialidad}

En gran medida, los estudios de la adaptación de los grupos cazadores-recolectores han focalizado su atención en la movilidad y los sistemas de 
asentamiento. En otras palabras, como los seres humanos han explotado uno de los recursos claves: el espacio. Willey (1953) fue el que 'popularizó' este tipo de acercamiento, al estudiar los patrones de asentamiento en el valle del Virú, Perú, pero no para grupos cazadores-recolectores. Willey exploró como los diferentes sitios arqueológicos se relacionaban con el asentamiento geográfico y el ambiente. Años más tarde, Winters (1969) introduce la diferencia entre patrón y sistema de asentamiento. Las relaciones geográficas y fisiográficas de un grupo de sitios pertenecientes a una 'cultura' son interpretados como el patrón de asentamiento, en tanto que las relaciones funcionales entre los sitios incluídos en un determinado patrón, son asumidos como el sistema de asentamiento.

No es hasta los trabajos de Vita Finzi y Higgs (1970) que el espacio comienza a ser estudiado en una forma más coherente - sistemática y metodologicamente considerados. La formulación del 'site catchment analysis' con sus pros y contras (ver Roper 1979 y Dennell 1980) coloca al arqueólogo en la necesidad de ver 'afuera' del sitio.

Alternativamente, Clark (1977) discute la importancia del espacio en sí mismo como recurso. Clark señala que el ambiente no está compuesto únicamente por la distribución de los recursos sino que también incorpora al espacio. Es importante reconocer que las poblaciones humanas no pueden ser estudiadas aisladamente y que los sistemas adaptativos se influencian y coactúan unos con otros. El espacio puede ser empleado por los grupos humanos para resolver problemas de competencia por recursos, incompatibilidad, movilidad, etc.. Esto es en líneas muy generales, el concepto de territorialidad.

Recientemente, se ha desarrollado una nueva y diferente perspectiva de asumir el registro arqueológico. Esta involucra la relación entre el registro arqueológico y el espacio, considerando al primero un continuum en el segundo, más que un desecho estático y/o sincrónico de la vida pasada (Foley, 1981; Dunnell y Dancey, 1983; Dunnell, 1988; Ebert, 1992; Borrero y Lanata, 1992; Belardi, 1991, etc.). La conceptualización del registro arqueológico como un continuum en espacio y tiempo remarca la importancia de los sitios de baja densidad de hallazgos - y aún de los hallazgos aislados - en la explicación de los sistemas de asentamiento. En otras palabras ver al registro arqueológico en una dimensión espacial, sin focalizaciones puntuales. Bajo esta perspectiva, el análisis del espacio no puede ser equiparado con el de los patrones de asentamiento.

La incorporación de nuevas perspectivas ecología, etnoarqueologia, biogeografia, etc.) y la adopción de enfoques evolutivos y ecológicos han contribuído a ver más la inter-relación entre los grupos humanos y los animales como relaciones predador-presa. Sin embargo, y en forma más general, las relaciones entre los cazadoresrecolectores - en nuestro caso - y los diferentes aspectos del ambiente, pueden ser comprendidas más adecuadamente, si las vemos como relaciones predador/consumidor-recurso. De esta manera, este simple modelo incluye todo tipo de recursos disponibles y/o empleados, no solamente los animales.

El control-uso del espacio, y de los recursos distribuídos en él es, de alguna manera, conducta territorial. Este concepto ha sido formalmente aplicado a poblaciones humanas por DysonHudson y Smith (1978). Los seres humanos como cualquier otra especie animal - tienen conductas territoriales. De alguna u otra manera definen y defienden un espacio, el cual es asumido como propio. Como Dyson-Hudson y Smith (1978) proponen, un territorio puede ser de interpretado como " ... an area occupied more or less exclusively by an individual or group by means of repulsion through overt defense or some form of communication". Sin embargo, debemos señalar que diferentes grupos frecuentemente comparten territorios. Espacios compartidos en forma cooperativa, religiosa, económica, etc. son comunes entre grupos que exhiben formas adaptativas complementarias o no, y ésto puede ocurrir igualmente entre grupos que ocupan diferentes nichos ecológicos (Jochin, 1983). También, estrategias de fusión-fisión (Thomas, 1983) pueden tener lugar en cualquier lugar y en cualquier momento entre grupos vecinos.

Los estudios etnoarqueológicos (Yellen, 1977; Binford, 1978; O’Connell y Hawkes, 1984; Smith, 1991, etc.) han contribuído, en gran medida, a clarificar la relación entre sistemas de asentamiento y territorialidad. Ellos han mostrado el grado de variabilidad, diversidad funcional y flexibilidad que puede existir en diferentes cirscunstancias (Williams 1982). Considerando 
LANATA, J. L. Evolución, espacio y adaptación en grupos cazadores-recolectores . Rev. do Museu de Arqueologia e Etnologia, S. Paulo, 3: 3-15, 1993.

estas variaciones, Dyson-Hudson y Smith (1978) adoptan la idea de Brown (1964) de defensa económica del espacio. El modelo de Brown sugiere que en un espacio defendido, el costo de defensa y uso del mismo es balanceado con los beneficios y ventajas relacionados con el control exclusivo sobre los recursos involucrados en ese espacio. "Economic defendability is determined by the interaction of foraging behavior and territorial defense with a particular distribution in space and time of critical resources" (DysonHudson y Smith 1978:23).

Para sugerir la defensa de un espacio en particular - es decir para un territorio-debemos considerar:

1) el tiempo y la energía colocada para controlar, proteger y mantener ese espacio

2) la estructura de los recursos; la distribución espacio temporal y la relación entre recursos disponibles y potenciales.

3) los beneficios, o sea la ganancia neta, en términos de fitness biológico y cultural, de mantener ese espacio.

Cuando la conducta territorial está presente, podemos esperar que los beneficios - 3) sean de mayor, o al menos igual, importancia que los costos - 1). Si los grupos explotan óptimamente su ambiente, la estructura de los recursos determina la relación entre outputs e imputs, y consecuentemente, la conducta territorial. Sin embargo, como en relación entre el rango de recursos disponibles y los recursos seleccionados es definido por el grupo y su toma de decisiones, la conducta óptima es muy dificil de ser asumida a priori.

Ambrose y Lorenz (1990:13) definen a la estructura de recursos como la relación entre cinco elementos claves: predictibilidad, distribución, densidad, disponibilidad y diversidad de los distintos recursos en una área determinada. Todos estos factores son interdependientes y varían en espacio y tiempo.

Para poder relacionar la estructura de recursos con un sistema de asentamiento es necesario examinar la disponibilidad y la selección de recursos efectuada a lo largo de un bloque espaciotiempo. Fluctuaciones y/o alteraciones en cualquiera de los cinco parámetros de la estructura de recursos pueden tener alguna correlación con la respuesta de un sistema adaptativo. Por ejemplo, la diversificación en la subsistencia ha sido frecuentemente sugerida como respuesta a un incremento en los costos de apropiación de los recursos claves. Movilidad, tamaño del grupo, emplazamiento de sitios y otras variables pueden ser calificadas e interpretadas por la apropiación de determinados recursos en tiempos especificos.

Los grupos humanos adoptan diferentes estrategias para defender el espacio por ellos explotado. Como Dyson-Hudson y Smith (1978) proponen, la defensa económica de un espacio es una de las bases más apropiadas para discutir el problema de la territorialidad. Pero no debemos descartar otros motivos para defender una determinada área. Sin embargo, debemos ser cautos y diferenciar entre la idea de territorio y el concepto de territorialidad.

Ambos términos son usados etnograficamente en arqueología. Con esto quiero decir que, usualmente, los arqueólogos argumentamos sobre territorios y territorialidad en una forma sistémica (sensu Schiffer, 1972). Como mencioné anteriormente, el territorio puede ser considerado como el espacio que los cazadores-recolectores defienden. Sin embargo, la territorialidad, como una conducta, puede cambiar a causa de diferentes factores.

Resumiendo, los sistemas de asentamiento armonizan diferentes estrategias, tácticas o rasgos (i.e. movilidad, tamaño del grupo, ubicación de los sitios, etc.) en una estrategia general. Los grupos humanos hacen operativas diferentes estrategias para establecer su relación con una estructura de recursos en un determinado bloque espacio-tiempo. Estas estrategias pueden variar con cualquier fluctuación o modificación de alguno de los recursos seleccionados. Consecuentemente, la selección de una estrategia conlleva la incorporación de algún grado de flexibilidad que balancee la posibilidad de fluctuaciones en los recursos. En otras palabras, el grado de flexibiliad está relacionado con la noción de riesgo y/o incertidumbre en relación con la disponibilidad de uno y/o varios recursos.

\section{Revisando a los 'forager-collectors'}

Usualmente, los estudios normativos de las adaptaciones de los cazadores-recolectores han polarizado sus caracterizaciones en modelos lineales generales. Esta conceptualización lineal supone que cualquier grado de variabilidad, como 
por ejemplo sedentariness, está representado en un punto dentro del continuo.

Woodburn (1982) propuso una clasificación de grupos cazadores recolectores de acuerdo con el tiempo utilizado y los inputs energéticos. La diferencia radica fundamentalmente entre aquellos que poseen estrategias de retorno inmediato y los que lo postergan. Issac (1978) y Gould (1981) proponen que uno de los puntos más destacados de los cazadores recolectores es la forma y la cantidad de prácticas de compartir alimentos, tareas, etc. Otra clasificación es la de Testart (1982) que diferencia a los grupos de acuerdo con el nivel de alimentos conservados. Bettinger y Baumhoff (1982) diferencian entre estrategias 'processor' y 'traveller'. Price y Brown (1985) y Keeley (1988) diferencian entre adaptaciones simples y complejas de cazadoresrecolectores. Pero sin duda, una de las más populares y controversiales clasificaciones es la Binford (1980).

Binford (1980) introduce la terminologia 'forager-collector' para explorar los sistemas de movilidad y la organización de los asentamientos de los cazadores-recolectores. La dicotomía 'forager-collector', ha sido considerada como representativa de grupos específicos. Por ejemplo, los Aché de Paraguay y los San de Botswana han sido identificados como 'foragers', en tanto que los Nunamiut como 'collectors'.

El modelo de Binford puede ser empleado en diferentes niveles, de manera tal que incorpore tanto las manifestaciones arqueológicas del sistemas de asentamiento como los particulares detalles del mismo. Estrategias 'forager' llevan consigo una alta movilidad residencial, con apropiación diaria de alimentos y una no intensiva explotacion de los recursos. Como registro arqueológico, las estrategias 'forager' pueden exibir muy poca actividad intra sitio y a una gran variabilidad inter sitio, resultante de los cambios en cada estación. Las actividades "off site" (sensu Foley, 1981) pueden ser también importantes.

Las estrategias 'collector' son diferentes. La movilidad residencial es menor. Los sitios poseen mayores actividades especificas y el mismo Binford (1980) argumenta que grupos de tarea especiales son comunes. Los diferentes sitios son diferenciados como bases residenciales, locaciones, 'field camps', estaciones y 'caches'. La reutilizacion de los sitios es frecuente durante el ciclo anual, y en ocasiones, con diferentes actividades.

Estas dos estrategias fueron formuladas para comprender los sistemas de asentamiento e interpretar el registro arqueológico de los grupos cazadores-recolectores. El continuum que ellas definen puede ser usado para predecir la estructura general del registro arqueológico en tiempo y espacio. Para estrategias 'forager', la densidad de artefactos esperada es baja con una distribución muy esparcida en toda la región. La frecuencia y variedad de tipos de sitios puede ser elevada. La diversidad de artefactos entre los diferentes conjuntos tiende a ser baja. Las estrategias 'collector' parecen producir conjuntos con alta densidad de desechos y una variabilidad de baja de tipos de sitios. Por el contrario, la diversidad artefactual es mucho mayor que entre las estrategias 'forager'.

La dicotomía 'forager-collector' puede ser aplicada al registro arqueológico. Patrones de movilidad, apropiación y repartición de alimentos, estacionalidad y cronograma de actividades así como cambios en la función de los sitios caracterizan las estrategias adaptativas. Y determinan la posición de un grupo dentro del 'forager-collector' continuum. Binford (1980) señala que los grupos 'forager' y 'collector' no son "two polar types" de sistemas de asentamiento y subsistencia opuestos, y que ellos pueden estar presentes "in the organization of a single society". De hecho, el modelo 'forager-collector' sólo pueder ser visto como una herramienta heuristica con la cual podemos estudiar semejanzas y diferencias entre sistemas. Nuestras interpretaciones se encuentran 'enturbiadas' por una amplia disposición de variables socio-politicas, económicas, ecológicas, ideológicas, etc.. Muchas de estas variables pueden ser - o son - ambigüas $y / o$ indecifrables arqueologicamente. Puede ser que in modelo multidmensional que involucre subsistencia, asentamiento, considere los inputs y outputs energéticos, territorialidad, competencia entre grupos, etc. pueda ser propuesto como sustituto del modelo forager-collector.

Uno de los problemas de adoptar un modelo dicotómico de los sistemas de asentamiento y subsistencia como medio para comprender el registro arqueológico, es que los grupos pueden estar reutilizando lugares - sitios - con diferentes funciones, estructuras, frecuencias, cantidad de personas, etc.. Como Ebert y Kohler (1988:114) 
señalan, las observaciones, tratando de buscar la reutilizacion de sitios, tienen “... important implications for our understanding of the complexity of the archaeological record". Obviamente, entender los cambios en la función de sitios es un prerequisito de la interpretación de cualquier sistema de asentamiento

La dicotomía (Tomka, 1990; Johnson, 1991, etc.) o el continuum (Bamforth, 1988; Ebert and Kolher; 1988, etc.) 'forager-collector' es, esencialmente, una aplicación de las generalizaciones etnoarqueológicas como un mecanismo interpretativo con el cual podemos producir explicaciones sistémicas del registro arqueológico (sensu Schiffer, 1976). En los casos de la etnoarqueología y etnografía, las variables espaciales y temporales se encuentran más o menos delimitadas; están espacialmente acotadas y son regularmente sincrónicas. En arqueología, es necesario producir una representación diacrónica bien balanceada de las adaptaciones de los cazadores-recolectores (Thomas, 1983) que refleje la naturaleza del registro arqueológico. El modelo 'forager-collector' requiere de adecuados controles temporales para ser aplicado al registro arqueológico. Si bien puede parecer simple su aplicación a sistemas de asentamiento conformados por sitios compuestos por ocupaciones más o menos simples, su aplicación a contextos del tipo multicomponente es tanto o más dificultoso.

Los registros etnográficos y etnoarqueológicos nos muestran que típicos 'forager', como los San, emplean estrategias 'collector' (Yelen, 1977). Los Selk'nam de Tierra del Fuego tienen estrategias de movilidad tipo 'forager', sin embargo actúan como 'collectors' cuando una ballena vara en alguna playa (Gusinde, 1982). Permanecen cerca del varamiento por varios meses, en forma independiente de la estación. Por el contrario, pastores Navajos cultivan maiz y cazan en lugares apartados del sitio de residencia (Russel, 1978), pudiendo producir un muy confuso y superpuesto sistema de asentamiento. Con estos sencillos ejemplos, es obvio que formas complejas y combinadas de estrategias de asentamiento y movilidad pueden tener lugar en cualquier tipo de grupo.

El uso de Tomka (1990), Johnson (1991) y el de Ebert y Kolher (1988), Bamforth (1988) - entre otros autores - del modelo de 'forager-collector' es sin duda, diferente. Tomka (1990) y Johnson (1991) asumen que las estrategias son puntos opuestos, en tanto que Ebert y Kolher (1988), Bamforth (1988) lo conciven como un continuo que va de lo 'forager' a lo 'collector'. Obviamente, cualquier simple subsistema relacionado con un simple recurso, como por ejemplo la localizacion óptima para la recolección de nueces puede exhibir tanto tácticas 'forager' o 'collector'. Sin embargo, como una sistema total - o aún como una estrategia - la adaptación sólo puede ser caracterizada en forma general, ya sea como primariamente 'forager', o del tipo 'collector'.

Si bien el objetivo de Binford al proponer la terminologia 'forager-collector' fue entender la variabilidad de los cazadores recolectores en relación a los sistemas de asentamiento, el presupuesto de un continuum lineal anula la comprehensión de la variabilidad intrasistémica. Por otra parte, la dicotomización del modelo oculta la posibilidad de una válida caracterización del sistema in toto, así como de sus componentes.

La teoria de la Depredación - Foraging Theory - (i.e. MacArthur y Pianka, 1966; Stephens y Charnov, 1982, etc.), en forma especial el modelo de ampitud de dieta, puede ser aplicado al modelo 'forager-collector'. J Cuanto tiempo de búsqueda y manejo es empleado en una estrategia 'forager' en un recurso dado? Parece obvio que las estrategias 'collector' sensu Binford - involucran un costo mayor en el manejo del mismo. Por otro lado, las estrategias 'forager' parecen insumir un tiempo mayor en la búsqueda del recurso. Las relaciones hombrerecurso pueden ser clasificadas en forma estática y sincrónica como 'forager' o 'collector'. Pero como muchos grupos poseen ambos tipos de estrategias, focalizar las relaciones entre grupos y un determinado recurso - i.e. guanaco, obsidiana, etc. - como componentes de un sistema total, no hace más que impedir un adecuado acercamiento conceptual al problema arqueológico.

\section{Cazadores recolectores, estructura de recursos y riesgo}

Los cazadores-recolectores han sido erróneamente considerados como poblaciones con perfectas - o semi perfectas - adaptaciones al ambiente (i.e. Sahlins, 1972). Este esquema simplísta de la adaptación humana no tiene en cuenta el hecho que las estrategias adaptativas 
LANATA, J. L. Evolución, espacio y adaptación en grupos cazadores-recolectores . Rev do Museu de Arqueologia e Etnologia, S. Paulo, 3: 3-15, 1993.

no significan necesariamente un exito adaptativo en el largo plazo (ver Dunnell, 1980). Siguiendo el esquema spenceriano de la evolución (ver comentarios en Rindos, 1984; O'Brien y Holland, 1992), la evolución cultural es vista como un continuo de simples a complejas formas $y / o$ procesos de adaptación. Esto sugiere una trayectoria lineal de la misma. Sin embargo, las conductas humanas pueden considerarse en la perspectiva arqueológioa sin la necesidad de asumir maximizaciones económicas y/o modelos unilineales de evolución (Dunnell, 1989).

Si asumimos que es posible aplicar en un sentido selectivo darwiniano (Dunnell, 1989) las estrategias de cazadores-recolectores y la Teoria de la Depredación al registro arqueológico, necesitamos moderar y conocer las propiedades del mismo. Para discutir de esta manera las estrategias adaptativas de grupos cazadoresrecolectores, se hace necesario considerar:

a) la estructura de los recursos

b) determinar adecuadas escalas analíticas temporo-espaciales

c) distribución del registro arqueológico

d) el grado de integridad del registro arqueológico en b)

e) diversidad del registro

f) la relación entre tamaño de la muestra y clases de artefactos presentes

g) la relación entre la densidad de artefactos y su distribución en el espacio

Como Mayr (1982) remarca la adaptación “...could no longer be considered a static condition". Los grupos viven en ambientes que son dinámicos y heterogéneos. Los seres humanos como cualquier otro organismo - cambian constamente para modificar su ajuste al medio. En un modo general, la adaptación opera para mantener una población estable por sobre los factores ambientales. Realizan diferentes selecciones (generales y/o particulares, en forman azarosas y no dirigidas) con la finalidad de asegurar su descendencia. Muchas de estas selecciones se pueden incluir como una sección del fenotipo humano, como lo son los artefactos (Dunnell, 1989; Lanata y Borrero, 1991; Smith, 1991; O'Brien y Holland, 1992). Y no todas estas selecciones son existosas necesariamente. En estas condiciones de cambio constante, debemos considerar que los cazadores-recolectores no viven en ambientes homogéneos. La mayoria de los ambientes (con algunas - posibles - excepciones como el desierto del Sahara, los Polos, etc.) tienen sus diferentes comunidades bióticas y abióticas distribuidas heterogenamente, es decir en patchs, con diferentes productividades en el tiempo.

Adoptar una perspectiva neo-darwiniana trae como consecuencia el uso de modelos de depredación para la interpretación del registro arqueológico (ver Bettinger, 1991 y ejemplos alli citados). Todos estos modelos de depredación son deterministicos (i.e. el teorema del valor marginal - Charnov, 1976), en el sentido que ellos se encuentran matematicamente construídos y generan expectativas concretas. Estos modelos determinísticos no tienen en cuenta posibles modificaciones azarosas y/o catastróficas (Stephens y Charnov, 1982) ni tampoco consideran variables que no sean ni tecnológicas ni ambientales en los sistemas.

Los cazadores-recolectores pueden maximinar el tiempo empleado en determinadas actividades - i.e. caza - en uno de los patchs, minimizando posibles grados de riesgos e incertidumbre. Hipoteticamente, el tiempo que los cazadoresrecolectores emplean en un determinado lugar debe relacionarse con la energía que del mismo captan o ganan. Como Stephen y Charnov (1982) señalan, los modelos estocásticos son importantes en el estudio de la conducta de depredación, ya que la mayoría de los fenómenos biológicos son estocásticos (Stephens y Charnov, 1982:251).

Tanto los modelos determinísticos como los estocásticos son útiles pero en diferentes maneras. Los modelos deterministicos lo son para predecir estrategias o establecer standars de comparación entre ellas. Desafortunadamente, también requieren como condición considerar al ambiente como estable. Los modelos estocásticos, tal cual lo proponen Stephens y Charnov (1982) son más informativos para modelar condiciones de variabilidad, ya que incorporan la selección de un rango de estrategias alternativas. Resumiendo, los modelos deterministicos de depredación pueden ser empleados con mayor éxito bajo condiciones de estabilidad en determinados bloques espaciotemporales, en tanto que los estocásticos parecen ser de mayor utilidad para el estudio de la variabilidad. 
La Teoría de la Depredación requiere ciertos presupuestos, tal como el concepto del central place (lugar central). Orians y Pearson (1979) asumen que 1) todo organismo tiene un lugar central y 2) to dos los patchs pueden ser explotados desde el mismo. Este concepto del lugar central asume que los individuos captan energía en lugares externos al lugar central de operaciones y luego regresan al mismo. El modelo del lugar central está condicionado por las cualidades de los patchs, la disponibilidad de los recursos, competencia y riesgo (Orians y Pearson 1979: 156). Este modelo incluye tanto diferentes alternativas de apropiación de la presa (simple o múltiple), como elección de patch e itinerarios óptimos.

Como Krebs (1979:226) señala, la teoria de la depredación está limitada a la consideración de costos y beneficios relacionados con recursos específicos, pero ignora como el depredador "allocates its time between foraging and another activities, and it also does not take into account the possibility that during a foraging bout, a predator might aim to achieve an alternative goal". Eventos fortuitos pueden interrumpir y modificar el programa programado previamente así como modificar la utilización de recursos. Claramente. el tiempo debe ser tratado también como un recurso.

Los sistemas de asentamiento de cazadoresrecolectores parecen tener una complejidad tal, diferente a la de otros animales depredadores. Por lo tanto, para aplicar Foraging Theory a grupos cazadores-recolectores humanos, es importante incorporar la idea del registro arqueológico como continuum tanto en el espacio (ver más arriba) como en el tiempo. Como Dunnell (1992:212) señala, 'the archaeological record is generated continously'. De esta forma, estamos recuperando dos mal entendidas y comprendidas variables del registro arqueológico, el tiempo y el espacio. Adoptando esta perspectiva, estamos en condiciones de discutir como ha sido su depositación, como es su diversidad, densidad, frecuencia y distribución.

En arqueologia, los modelos estocásticos nos ayudan a entender riesgo e incertidumbre (i.e. de Garine y Harrison, 1988, y artículos, Cashdan, 1990a y artículos). Las ideas de riesgo e incertidumbre derivan de la economía, donde es aplicada fundameantalmente en la toma de decisiones (Lattimore, 1988). En microeconomía, riesgo e incertidumbre son polarizados en puro riesgo y pura incertidumbre (Hey, 1979). En ecología cultural, incertidumbre pura es el resultado de la aparición de problemas fortuitos o la carencia de información de la estructura de recursos necesaria de forma tal que se inhibe la selección de decisiones.

Recientemente, Cashdan definió riesgo como "unpredictable variation in some ecological or economic variable (1990b:2). Sin embargo, esta definición de riesgo es claramente contingente y conceptualmente semejante con la noción de incertidumbre. A mi entender, ambos conceptos parecen ser indistinguibles arqueologicamente.

La idea de riesgo se encuentra ligada a la de utilidad (Stephens y Charnov, 1982:259). Y utilidad está definida por la cantidad de un recurso específico y su posición en el ranking de outcomes disponibles en una población. Los cazadores-recolectores deben "relate behavior to the distribution of some intermediate reward (e.g. amount of food). The expectation of the utility function (...) provides the final link to the currency" (Stephens y Chamov, 1982:259). Stephens (1990) y Winterhalder (1990) ven al riesgo "... as the probability of falling below some minimum level of income of food intake" (Cashdan, 1990b:3). En ambas condiciones, riesgo está asociado con la estructura de recursos disponible.

Gould y Yellen (1987 y 1991) y Binford (1991) también utilizan los conceptos de riesgo e incerti-dumbre. Ellos discuten como la presencia de predadores cerca de los campamentos pueden causar una determinada forma en la estructura del household. Este punto de vista de riesgo e incertidumbre es muy diferente al de Stephens (1990), Winterhalder (1990), Cashdan (1990), etc. Si asumimos que la estructura de un household está determinada por la presencia/ausencia de predadores, ésta debe ser considerada como una constante, no como una variable azarosa. Desde un punto de vista arqueológico, es mucho mejor el uso de los conceptos de riesgo e incertidumbre para discutir modificaciones contingentes en, i.e. la disponibilidad de recursos, que en casos peligro en donde la situación es predecible por parte del grupo y/o los individuos.

Cuando cambios ambientales - sin importar su magnitud - producen modificaciones en la estructura de los recursos disponibles, la territorialidad puede también cambiar. Como Dyson-Hudson y Smith (1978) proponen, la 
defensa económica de un espacio es una de las formas más apropiadas con las que nos podemos aproximarnos al estudio de las conductas territoriales. Territorialidad es definida como el sistema de conductas que controlan y mantienen el uso más o menos exclusivo sobre un área específica. Tanto territorio como territorialidad pueden cambiar en respuesta a diferentes estímulos.
Uno de los legados de la etnografia, con el cual la arqueología sigue luchando, es la idea de frontera o limite. Ambrose y Lorenz (1990: 13-18), con una perspectiva ecológica, describen al menos tres tipos diferentes de límites: a) defendidos, b) no defendido pero frecuentemente cruzado $y \mathrm{c}$ ) abierto, donde diferentes grupos, recursos e información se encuentran.

\section{Leyenda}

ÓPTIMO

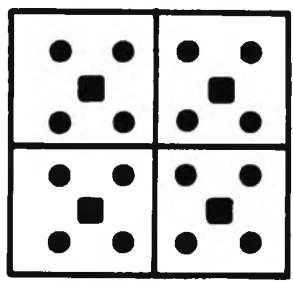

A. Previsible y denso

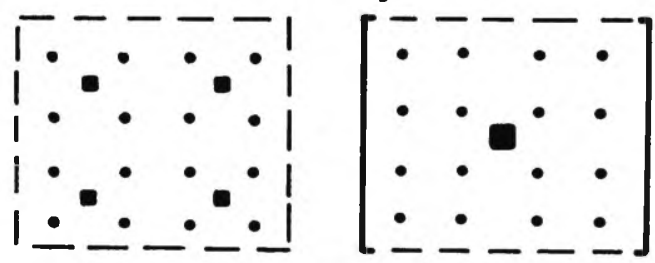

B. Previsible y raro

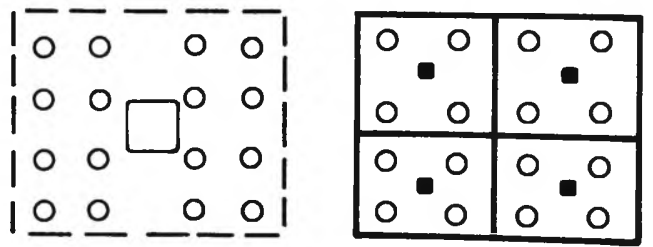

C. Imprevisible y denso
Asentamientos inestables, grupo de tamaño grande, muy alta mobilidad

Asentamientos inestables, grupo de tamaño pequeño, muy alta mobilidad

Frontera territorial defendida

Frontera de alcance doméstico no defendida, esporadicamente atravesada

Frontera abierta y no defendida a través de la cual flue información, recursos, personas y grupos

$\begin{array}{lllll:lllll}\Delta & \Delta & \Delta & \Delta & \Delta & \Delta & \Delta \\ \Delta & \Delta & \Delta & \Delta & \Delta & \Delta & \Delta \\ \Delta & \Delta & \Delta & \Delta & \Delta & \Delta & \Delta & \Delta \\ 0 & \Delta & \Delta & \Delta & \Delta & \Delta & \Delta\end{array}$

D. Imprevisible y raro

Figura 1 - Modelos óptimos y sub-óptimos de organización socio-territorial, según Ambrose y Lorenz, 1990:15- Figura 1.2 
LANATA, J. L. Evolución, espacio y adaptación en grupos cazadores-recolectores . Rev. do Museu de Arqueologia e Etnologia, S. Paulo, 3: 3-15, 1993.

Los cazadores-recolectores pueden poseer una territorialidad diferente a lo largo de las distintas estaciones y/o momentos del año. Muchos de los cambios en la territorialidad pueden relacionarse con la estructura de los recursos. Estas relaciones entre la estructura de recursos y los límites en los territorios han sido estudiadas por Ambrose y Lorenz (1990). Estos autores proponen ocho diferentes modelos (óptimos y subóptimos) que llaman estrategias de organización socio-territorial (socio-territorial tiempo es una importante variable ya que un sistema de asentamiento de cazadores-recolectores, y su contexto ambiental, cambian. En la figura № 2 presentamos una hipotética variación espacio-temporal en los límites de un grupo cazador-recolector, de acuerdo con los cuatro posibles tipos de límites.

Un simple análisis de la figura citada nos muestra el grado de variabilidad de un territorio en relación con las diferentes territorialidades exhibidas por un determinado grupo. La

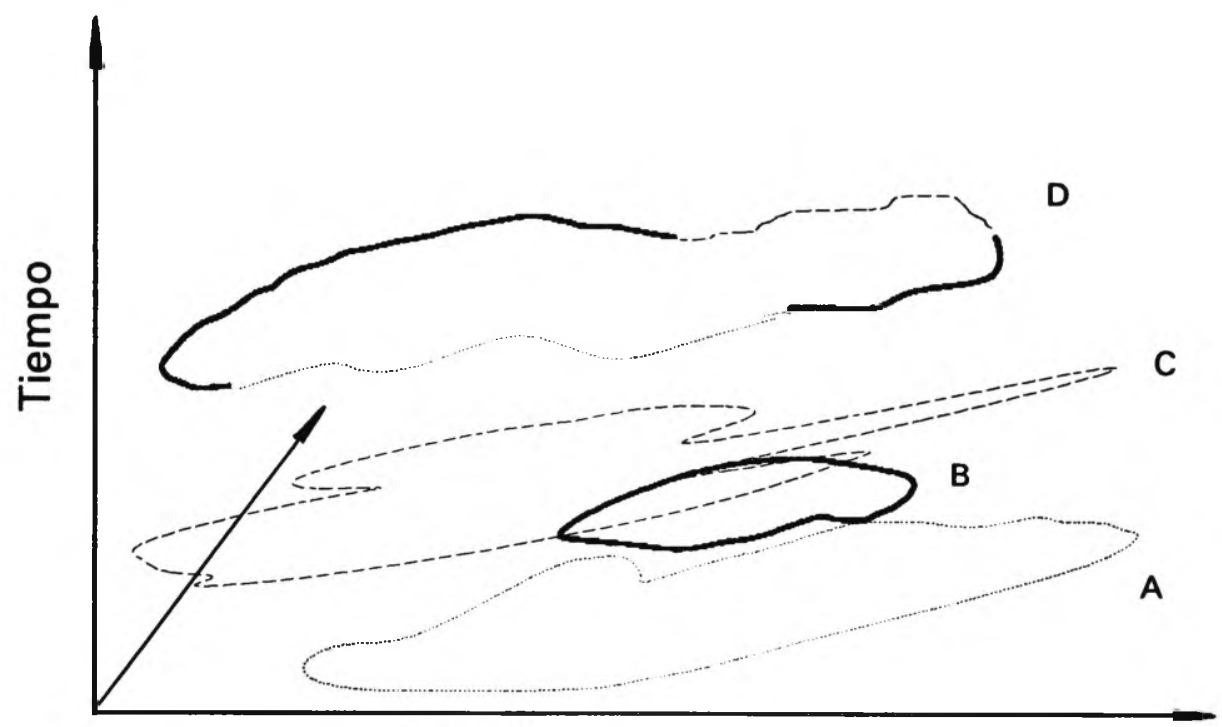

\section{Espacio}

Tipo de límite : $\quad$ defendido

no defendido

abierto

Fig. 2 - Variación espacio-temporal en los limites de um grupo cazador-recolector.

organizational strategy) (ver figura №1).

Además de los tres tipos de limites ya mencionados, proponemos un cuarto caso en dónde los tres tipos de fronteras son utilizados simultaneamente. De ello también derivarian conductas territoriales diferentes.

Las relaciones entre los límites y sus posibles combinaciones implican sistemas estáticos y sincrónicos. Una consideración de la variabilidad temporal lleva como consecuencia colocarlas en una perspectiva más diacrónica, y por lo tanto, mucho más aplicable arqueológicamente. El visualización arqueológica es aún más compleja si consideramos a) los procesos de formación del registro y b) su distribución espacio-temporal.

\section{Comentarios generales y consideraciones}

A lo largo de este trabajo hemos presentado diferentes aspectos que están siendo empleados en los enfoques evolutivos. Hemos tratado de hacerlo de acuerdo a las diferentes perspectivas de los autores. Pasaremos a continuación a considerar y comentar aspectos que creemos de 
suma importancia para la investigación arqueológica, siempre desde una visión evolutiva.

Los enfoques evolutivos citados podemos dividirlos en aquellos que han tratado con aspectos de la ecología evolutiva (Stephens y Krebs, 1986; Smith y Winterhalder, 1992, etc.) y los que han tratado una aproximación arqueológica (O'Brien y Holland ,1990 y 1992; Dunnell, 1992; Ebert, 1992 , etc.).

Desde el punto de vista de la idea de territorio aplicada al registro arqueológico, muchos trabajos han usado este concepto en forma acrítica. Desde la incorporación de 'site catchment analysis' (Vita Finzi y Higgs, 1970) en adelante, la noción de territorio bosquejado arqueologicamente a partir de un sitio, trajo consigo la idea de necesaria relación de defensa - por no decir territorialidad - entre el área circundante a un sitio y los ocupantes del mismo - ver definición de territorio en Higgs y Vita Finzi, 1972. Es aquí donde la variabilidad en tipos de límites citada anteriormente, cobra importancia.'

Un grupo posee diferentes tipos de fronteras a lo largo del tiempo en que explota un espacio. De esta manera, los residuos arqueológicos presentan distribuciones que fluctúan en relación con los tipos de límites empleados. El grado de resolución del registro arqueológico es, por el momento, de grano grueso. Los límites espacio temporales que podemos establecer son, en consecuencia, amplios. Los estudios derivados de la ecología evolutiva, y también de la etnoarqueología, nos están mostrando una serie de propiedades que pueden ser de aplicabilidad en el estudio arqueológico, pero que nos llevan a niveles de resolución sistémicos (sensu Schiffer, 1972), inadecuados para las propiedades del registro con el que trabajamos. La noción de territorio - y más aún - de territorialidad en cazadores-recolectores, se torna inapropiada cuando el grado de resolución de los datos de una región es de centenares de años.

En los últimos años, los conceptos de riesgo e incertidumbre comenzaron a aplicarse a proble-

(1) El empleo de sistemas computarizados de análisis espaciales (i.e. Geographical Information System (GIS) con los diferentes tipos de programas disponibles - Idrisi, Archinfo, Atlas, etc.) pueden producir la proliferación del empleo inadecuado de análisis poco o nada relevantes para el estudio arqueológico. mas antropológicos. Desde la visión arqueológica, estos conceptos nos ayudan a entender mucho mejor una serie de selecciones que los grupos pueden realizar. Retomando el caso de los límites, riesgo y/o incertidumbre sobre determinados factores - i.e. inestabilidad ambiental, ausencia de recursos, etc. - son factores que repercuten en la decisión de un grupo al seleccionar alguno de los diferentes tipos mencionados.

El uso del modelo 'forager-collector' de Binford (1980) podemos analizarlo desde dos perspectivas. Una es el de asumirlo como un modelo general de adaptaciones, a través del cual podemos obtener un sinnúmero de posibles variantes y/o combinaciones. Otra es el de visualizarlo como estrategias relacionadas con recursos particulares, dentro de sistemas que nos permanecen más o menos desconocidos. La primera de las posibilidades es de neta aplicación actualística. El modelo 'forager-collector' - que no es más que una aplicación antropológica del teorema de la amplitud de dieta (ver MacArthur y Pianka, 1966) - necesita aún de adecuados conocimientos de las propiedades de depositación y descarte de artefactos a lo largo del tiempo y el espacio para poder asumirlos como posibles sistemas, siempre en términos del registro arqueológico. Asumir que un determinado registro arqueológico, con límites espaciales y temporales adecuados, pertenece un sistema adaptativo 'forager' o 'collector' nos impide conocer el grado de variabilidad del mismo. Por otra parte, y como nos señalara Dunnell (com. pers., 1992), no existe un lugar en los enfoques evolutivos para los 'sistemas adaptativos'. Estos no son más que sustituciones funcionales de los términos 'cultura', 'fase', 'tradición', etc. empleados por enfoques normativos, historicistas y/o históricos culturales.

La segunda de las posibilidades, es decir asumir el modelo 'forager-collector' como simples estrategias - no estrategias adaptativas - en lugar de sistemas adaptativos, parece ser más adecuado a la naturaleza del registro arqueológico. Las propiedades del mismo permiten una resolución que puede parecer más baja, pero que sin duda es mucho más explicativa de cambios en la distribución, la densidad, y la diversidad en espacio y tiempo de los materiales. La aplicación de modelos estocásticos y/o deterministas son de suma utilidad para 
comprender la variabilidad, siempre y cuando tengamos acotados los factores limitantes que mencionaramos más arriba.

\section{Palabras finales y puntos de partida}

Hemos presentado aqui, muy someramente, algunos de los principales tópicos de los enfoques evolutivos relacionados con el uso del espacio. De ello surge que podemos aproximamos al problema arqueológico desde el punto de vista de dos principales corrientes evolutivas. Una deriva de la ecología evolutiva (ver Smith y Winterhalder, 1992 y bibliografia alli citada; Foley, 1992) y otra que considera a los artefactos como parte del fenotipo humano (O'Brien y Holland, 1992; Dunnell, 1992, etc.). La primera se está dedicando al estudio de la evolución humana a través de las relaciones sociales, el cambio de las conductas, etc. La segunda ve el cambio a través de las propriedades del registro arqueológico. Estas dos posiciones nos son de suma utilidad, pero en dos niveles diferentes de nuestra investigación. La primera puede ser utilizada en un nivel interpretativo y general, en tanto que la segunda, lo es en una forma explicativa de la naturaleza del registro arqueológico. La integración entre ambas, y su aplicabilidad al problema de la evolución de los cazadoresrecolectores es, sin duda, un nuevo punto de partida.

\section{Agradecimientos}

La discusión de estos tópicos con Luis $\mathrm{A}$. Borrero, C. Britt Bousman, Robert Dunnell, James Ebert y Matt Tomasso, asi como los comentarios recibidos me han sido de mucha utilidad. El presente trabajo fue posible gracias a un subsisdio recibido del Consejo Nacional de Investigaciones Científicas y Técnicas, Argentina.

LANATA, J.L. Evolution, space and adaptation in hunter-gatherers groups. Rev. do Museu de Arqueologia e Etnologia, S. Paulo, 3: 15, 1993.

ABSTRACT: The use of space among hunter-gatherers and the relation with the general adaptation and evolution is discussed in this paper. Archaeologists often view the distribution in space of similar artifacts as pieces of human territories, without any kind of methodological frame that supports it. The ethnographic view in the explanation of the archaeological record is hiddenin the variability of human response to the different environment (natural, social, etc.).This paper shows that the idea of territory in archaeology needs to be settled aside and use a more consistently approach that matches more accurately the archaeological record properties.

UNITERMS: Spacial analysis - Hunter-gatherers - Adaptation.

\section{Bibliografia}

AMBROSE, S. H. \& K. G. LORENZ (1990) Social and ecological models for the Middle Stone Age in Southern Africa. The Emergence of Modern Humans. An archaeological perspective. Cornell University Press, thaca, New York:3-33.

BAMFORTH, D. B. (1988) Ecology and Human Organization on the Great Plains. Plenum Press, New York.
BELARDI, J. B. (1991) De lo espacial a lo temporal explorando distribuciones de artefactos en Cerro Castillo, Chubut. Trabajo presentado a la Revista de Estudios regionales. Universidad de Cuyo.

BETTINGER, R. L. (1980) Explanatory-predictive models of hunter-gatherer adaptation. Advances in Archaeological Method and Theory, 3:189-255. 
LANATA, J. L. Evolución, espacio y adaptación en grupos cazadores-recolectores. Rev. do Museu de Arqueologia e Etnologia, S. Paulo, 3: 3-15, 1993.

- (1991) Hunter-Gatherers. Archaeological and Evolutionary Theory. Plenum Press, New York.

BETTINGER, R. L.; M. A. Baumhoff, M.A. (1982) The Numic spread: Great Basin cultures in competition. American Antiquity, 47:485-503.

BINFORD, L. R. (1977) General Introduction.L.R. Binford (Ed.) For Theory Building in Archaeology. Academic Press, New York: 1-10.

- (1978) Nunamiut Ethnoarchaeology. Academic Press, New York

- (1980) Willow smoke and dogs' tail: hunter-gatherer settlement systems and archaeological site formation. American Antiquity, 45:4-20.

- (1991) Is australian site structure explained by the absence of predators? Journal of Anthropological Archaeology, 10:255-282.

BORRERO, L. A.; LANATA J. L. (1992) Arqueologia espacial en Patagonia: nuestra perspectiva. L.A. Borrero y J.L. compiladores. Análisis Espacial en la Arqueologia Patagónica. Búsqueda Ediciones, Buenos Aires: 145162.

BROWN, J. L. (1964) The evolution of diversity in avian territorial systems. Wilson Bulletin, 76:160-169.

CASHDAN, E. (1990a) Risk and Uncertainty in Tribal and Peasant Economies. Westview Press, Boulder.

- (1990b) Introduction. E. Cashdan (Ed.) Risk and Uncertainty in Tribal and Peasant Economies. Westview Press, Boulder: 1-16.

CHARNOV, E. L. (1976) Optimal Foraging: The manginal value theorem. Theoretical Population Biology, 9:129-136.

CLARKE, D. L. (1968) Analytical Archaeology. Methuen, London.

- (1977) Spatial information in archaeology. D.L. Clarke (Ed.) Spatial Archaeology. Academic Press, London: 132.

DE GARINE, l.; HARRISON, G.A. (Eds.) (1988) Coping with Uncertainty in Food Supply. Clarendon Press, Oxford.

DUNNELL, R. C. (1980) Evolutionary theory and archaeology. Advances in Archaeological Method and Theory, 3:35-99.

- (1989) Aspects of the application of evolutionary theory in archaeology. C.C. Lamberg-Karlovsky (Ed.) Archaeological Thought in America. University of Cambridge Press, Cambridge: 35-49.

- (1988) Low density archaeological record from plowed surfaces: some preliminary considerations. American Archaeology, 7:29-38.

(1992) The notion site. J. Rossignol and L. Wandsnider (Eds.) Space, Time and Archaeological Landscapes. Plenum Press, New York: 21-41.

DUNNELL, R. C.; Dancey, W.S. (1983) The siteless survey: A regional scale data collection strategy. Advances in Archaeological Method and Theory, 6:267-287.

DYSON-HUDSON, R.; Smith, E.A. (1978) Human territoriality: An ecological reassessment. American Anthropologist, 80: 21-41.
EBERT, J. I. (1992) Distributional Archaeology. University of New Mexico Press, Albuquerque.

EBERT, J. I.; KOHLER, T.A (1988) The theoretical basis of archaeological predictive modeling and a consideration of appropriate data-collection methods. W. James Judge and Lynne Sebastian (Eds.) Quantifying the Present and Predicting the Past: Theory, Method and Application of Archaeological Predictive Modeling. U.S. Department of the Interior, Bureau of Land Management, Denver, Colorado: 97-171.

FLANNERY, K. V. (1977) Archaeology with a capital "S". C.L. Redman (Ed.) Research and Theory in Current Archaeology. Wiley and Sons, New York: 47-53.

FOLEY, R. A. (1981) Off site archaeology: an alternative approach for the short sites. I. Hodder, G. Isaac and N. Hammond (Eds.) Pattern of the Past Studies in Honor of David L. Clarke. Cambridge University Press, Cambridge:157-183.

- (1992) Evolutionary Ecology of Fossil Hominids. E. A. Smith \& B. Winterhalder (Eds.) Evolutionary Ecology and Human Behavior. Aldine de Gruyter, New York: 131-164.

GOULD, R. A.; Yellen, J. E. (1987) Man the hunted determinance of household spacing in desert and tropical foraging societies. Journal of Anthropological Archaeology, 6:77-103

- (1991) Misreading the past: a reply to Binford concerning hunter-gatherer site structure. Journal of Anthropological Archaeology, 10:283-298

GRAYSON, D. K. (1986) Eoliths, archaeological ambiguity and the generation of "middle-range" research.D.J. Meltzer and D.D. Fowler (Eds.) American Archaeology: Past and Present, Smithsonian Institution Press. Washington, D.C: 73-133.

GUSINDE, M. (1982) Los Indios de Tierra del Fuego. Los Selk'nam. CAEA-CONICET. Buenos Aires, Argentina.

HEY, J.D. (1979) Uncertainty in Microeconomies. New York University Press, New York.

HODDER, I. (1986) Reading the Past: Current Approaches to Interpretation in Archaeology. Cambridge University Press, Cambridge.

ISAAC, G. L. (1978) Food sharing behavior of protohuman hominids. Scientific American, 238(4):90-108.

JOCHIM, M. (1979) Breaking down the systems: recent ecological approaches in archaeology. Advances in Archaeological Method and Theory, 2:77-117.

- (1983) Optimization models in context. A.S. Keene \& J. A. Moore (Eds.) Archaeological Hammers and Theories. Academic Press, New York: 157-172.

JOHNSON, E. (1991) Late pleistocene cultural occupation on the Southern Plains. R. Bonnichsen and K. Turnmire (Eds.) Clovis: Origins and Adaptations. Peopling of the Americas Publications, Oregon State University, Oregon: 215-236.

KEELEY, L. (1988) Hunter-gatherer economic complexity and "Population Pressure": a cross-cultural analysis. Journal of Anthropological Archaeology, 7:373:411. 
LANATA, J. L. Evolución, espacio y adaptación en grupos cazadores-recolectores . Rev. do Museu de Arqueologia e Etnologia, S. Paulo, 3: 3-15, 1993.

KREBS, J. R. (1979) Foraging strategies and their social significance. Peter Marler and J.G. Vandenbergh (Eds.) Handbook of Behavioral Neurobiology. Vol. 3. Social Behavior and Communication. Plenum Press, New York: 225-270.

LANATA, J.L.; BORRERO, L.A. (1991) Danza con lobos marinos. La relación hombre-pinnipedo en el extremo sur de América. A. Lichter (Ed.) Huellas en la arena, sombras en el mar. En prensa.

LATTIMORE, P. K. (1988) An Empirical Assessment of Alternative Models of risky decision making. National Bureau of Economic Research, Cambridge, MA.

MACARTHUR, R. H.; PIANKA, E.E. (1966) On optimal use of patchy enviromment. American Naturalist, 100:603-609.

MAYR, E. (1982) The Growth of Biological Thought. Diversity, Evolution and Inheritance. Harvard University Press, Massachusetts.

O'BRIEN, M.; HOLLAND, T.D. (1990) Variation, selection and the archaeological record. Archaeological Method and Theory, 2:31-79.

- (1992) The role of adaptation in archaeological explanation. American Antiquity, 57:36-59.

O'CONNELL, J. F.; HAWKES, K. (1984) Food choice and foraging sites among the Alyawara. Journal of Anthropological Research, 40:504-435.

ORIANS, G. H.; PEARSON, N.E. (1979) On the theory of central place foraging. D.J. Horn, G.R. Stairs and R.D. Mitchell (Eds.) Analysis of Ecological Systems. Ohio State University Press, Columbus: 155-177.

PRICE, T. D.; BROWN, J.A. (Eds.) (1985) Prehistoric Hunters-Gatherers: The emergence of cultural and social complexity. Academic Press, New York.

RAAB, L.M.; GOODYEAR, A.C. (1984) Middle - Range Theory in archaeology: a review of origins and applications. American Antiquity, 49:255-268.

RINDOS, D. (1984) The Origins of Agriculture: An Evolutionary Perspective. Academic Press, New York.

RUSSELL, S. C. (1978) The agricultural field house: a Navajo limited occupation and special use site. Albert $\mathrm{E}$. Ward (Ed.) Limited Activity and Occupation Sites: $A$ collection of Conference Papers, edited by Contribution to Anthropology $N^{\circ} 1$. Center of Anthropological Studies, Albuquerque: 35-40.

SAHLINS, M. (1972) Stone Age Economies. Aldine, Chicago.

SCHIFFER, M.B. (1972) Archaeological context and systemic context. American Antiquity, 37:156-165.

- (1987) Formation Processes of the Archaeological Record. University of New Mexico Press. New Mexico.

- (1988) The Structure of Archaeological Theory. American Antiquity, 53:461-485.

SMITH, E. A. (1991) Inujuamiut Foraging Strategies. Evolutionary Ecology of an Arctic Hunting Economy. Aldine de Gruyter, New York.
SMITH, E. A.; WINTHERALDER, B. (1981) New perspectives on hunter-gatherer socioecology. B. Wintherhalder and E. A. Smith (Eds.) Hunter-Gatherer Foraging Strategies. Ethnographic and Archaeological Analy. ses. The University of Chicago Press, Chicago: 1-12.

- (1992) Evolutionary Ecology and Human Behavior. Aldine de Gruyter, New York.

STEPHENS, D. W. (1990) Risk and incomplete information in behavioral ecology. E. Cashdan (Ed.) Risk and Uncertainty in Tribal and Peasant Economies. Westview Press, Boulder: 19-46.

STEPHENS, D. W.; CHAMOV, E.L. (1982) Optimal foraging: some simple stochastic models. Behavioral Ecology and Sociobiology, 10:251-263.

TESTART, A. (1982) The significance of food storage among hunter-gatherer: residence patterns, population densities and social inequality. Current Anthropology. 23:523-537.

THOMAS, D. H. (1983) The Archaeology of Monitor Valley. 1. Epistemology. Anthropological Papers of the American Museum of Natural History, 58(1), New York.

- (1986) Contemporary hunter-gatherer archaeology in America. D.J. Meltzer and D.D. Fowler (Eds.) American Archaeology: Past and Present. Smithsonian Institution Press, Washington D.C.: 237-276.

- (1989) Archaeology. Holt, Rinehart and Winston. Forth Worth.

TOMKA, S. A. (1990) Models of late Holocene human adaptation in the Lower Rio Grande Valley. Prehistoric Archaeology and Paleoenvironments in Hidalgo and Willacy Counties, South Texas: Results of the Phase II Test Excavations, by C. Britt Bousman, Steve A. Tomka and Gail L. Bailey. Reports of Investigations Number 76, Prewitt and Associates, Inc., Austin: 25-44.

VITA FINZI, C.; HIGGS, E. (1970) Prehistoric economy in the Mount Carmel of Palestine: site catchment analysis. Proceedings of the Prehistoric Society, 36:1-37.

WILLEY, G. (1953) Prehistoric Settlement Patterns in the Virú Valley, Peru. Bureau of American Ethnology, Bulletin 155. Washington D.C.

WINTERHALDER, B. (1990) Open field, common pot: harvest variability and risk avoidance in agriculture and foraging societies. E. Cashdan (Ed.) Risk and Uncertainty in Tribal and Peasant Economies, Westview Press, Boulder: 67-87.

WINTERS, J. (1969) The Riverton culture. A second millennium occupation in the Central Wabash Valley. Illinois State Museum. Report of Investigations $\mathrm{N}^{\circ} 13$.

WOODBURN, J. (1982) Egalitarian societies. Man, 17:431-451.

YELLEN, J. E. (1977) Archaeological Approaches to the Present: Models for reconstruing the Past. Academic Press, New York. 